\title{
Essais
}

\section{Approche matérielle des espaces réservés à la mort et aux morts}

Étude de l'arbre de l'Oubli et de l'arbre du Retour à Ouidah au Bénin

A material approach to spaces reserved for death and the dead Study of the tree of oblivion and the tree of return in Ouidah, Benin

\section{Carole Fagadé}

\section{(2) OpenEdition}

Journals

Édition électronique

URL : https://journals.openedition.org/essais/8705

DOI : 10.4000/essais.8705

ISSN : 2276-0970

Éditeur

École doctorale Montaigne Humanités

Édition imprimée

Date de publication : 15 avril 2021

Pagination : 87-96

ISBN : 979-10-97024-09-3

ISSN : $2417-4211$

Référence électronique

Carole Fagadé, "Approche matérielle des espaces réservés à la mort et aux morts », Essais [En ligne], 17 | 2021, mis en ligne le 27 avril 2021, consulté le 18 janvier 2023. URL : http:// journals.openedition.org/essais/8705; DOI : https://doi.org/10.4000/essais.8705

Ce document a été généré automatiquement le 18 janvier 2023.

Tous droits réservés 


\title{
Approche matérielle des espaces réservés à la mort et aux morts
}

\author{
Étude de l'arbre de l'Oubli et de l'arbre du Retour à Ouidah au Bénin
}

A material approach to spaces reserved for death and the dead Study of the tree of oblivion and the tree of return in Ouidah, Benin

\section{Carole Fagadé}

1 S'il existe dans l'histoire du Bénin et à Ouidah une porte du Non-Retour qui symbolise pour les esclaves noirs le début d'une vie entièrement dévouée à l'esclavage, il existe aussi un arbre du Retour qui symbolise le retour de l'esprit de ces esclaves, une fois qu'ils sont décédés à l'étranger. En effet, selon les croyances indigènes de Ouidah, même si l'individu a procédé avant son départ au rituel de l'Oubli, destiné à lui faire oublier ses « racines ", l'esprit de cet homme ne pourrait être supprimé. Ainsi, lorsque l'esclave meurt en terre étrangère, son esprit est susceptible de revenir sur la terre de ses ancêtres et l'arbre du Retour a pour rôle d'abriter son esprit et de l'immortaliser. Comme l'avait souligné Loraux, Nicole (1997), « Le non-oubli est un fantôme $»^{1}$.

2 Notre objectif est de montrer comment les esclaves instituaient leur propre «mort » avant qu'ils ne soient réellement morts. Nous voudrions montrer comment ces esclaves instituaient leur Retour. En un mot, il s'agit de comprendre comment, autour de l'esclavage, se construisent des pratiques liées à la mort. Comment sont-elles instituées? Notre intention est également d'étudier l'évolution des idéologies sur deux objets aussi incontournables que sont l'arbre de l'Oubli et l'arbre du Retour dans l'histoire du Bénin. De ce fait, nous avons questionné les usages sociaux, les modes d'appropriation et de communication qui participent à la construction de ces espaces aujourd'hui.

\section{L'arbre de l'Oubli et l'arbre du Retour}

3 Le Bénin, autrefois appelé royaume de Dahomey, fut l'un des principaux fournisseurs d'esclaves pendant la traite négrière. Au Bénin, l'esclavage a commencé dans la 
première moitié du XVII siècle. Au siècle suivant, précisément en 1750 , on estima les revenus du roi Tegbessou, l'un des principaux rois du pays, à quatre ou cinq fois plus élevés que ceux des propriétaires terriens d'Angleterre les plus influents². L'activité principale de ce roi était la vente des esclaves.

Aujourd'hui, l'histoire de l'esclavage au Bénin est essentiellement marquée par la « Route de l'Esclave » à Ouidah, la ville historique. Il faut entendre par cette expression non pas un simple chemin mais un "tracé douloureux", emblématique et métaphorique ${ }^{3}$ qui regroupe un ensemble de lieux, d'objets culturels et de rituels. La Route de l'Esclave s'étale sur trois kilomètres environ. Elle s'étale du centre-ville de Ouidah jusqu'à la plage, lieu où les esclaves étaient déportés par des navires pour servir dans des champs d'exploitation étrangers sous l'ordre de leur maître. La «Route de l'Esclave » est composée de six lieux que sont :

1. la place aux enchères (ou la place Chacha),

2. l'arbre de l'Oubli,

3. la case Zomaï (« que la lumière ne s'y approche »)",

4. le mémorial du village de Zoungbodji,

5. l'arbre du Retour,

6. la porte du Non-Retour.

4 Chacun de ces lieux servait à des rituels spécifiques. Mais seul l'arbre de l'Oubli et l'arbre du Retour nous intéressent dans cette étude. Ce qui est appelé l'arbre de l'Oubli ici n'est pas tout à fait un arbre. Il s'agit d'un monument représentant la divinité Mami wata. Celle-ci est considérée comme la mère des eaux. Cette divinité symbolise aussi bien la mère nourricière que la mère destructrice. Autour de ce monument, les esclaves femmes faisaient sept tours alors que les esclaves hommes en faisaient neuf. Ce passage obligatoire devrait les rendre amnésiques. 
Image 1 : L'arbre de l'Oubli

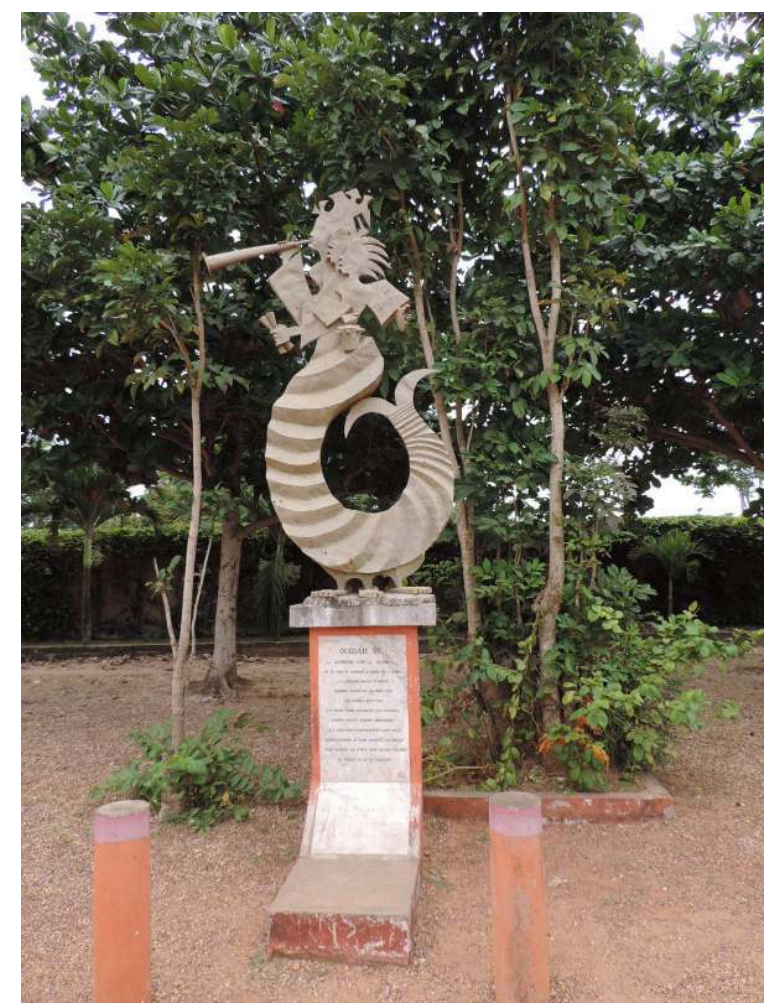

Source : https://fr.wikipedia.org/wiki/Fichier:Arbre_de_I_oubli_ouidah_benin.jpg

5 Qu'en est-il de l'arbre du Retour? Il s'agit précisément d'un arbre connu sous le nom scientifique de kigelia africana. L'arbre a été planté par le roi Agadja (1673-1740) sur la grande place appelée Zoungbodji, en 1727. Mais il n'est pas question de n'importe quel arbre, car cet arbre a été planté sur la dépouille de deux femmes et de deux hommes. Ces hommes et ces femmes furent des ennemis tués par le roi lui-même à partir d'incantations pendant la guerre d'expansion de son royaume qui l'a opposé au roi Houfon (Xufón, Xufon), roi des Pédah (Xwédah) en 1727. Ainsi, pour symboliser sa victoire, le roi décida de planter un arbre sur ces «ennemis » vaincus et ensevelis. L'arbre avait dès lors une image symbolique pour le royaume.

6 C'est bien plus tard que le prince Kakanakou, le fils du roi Agadja, suggéra à son père de faire faire aux esclaves vendus un "geste-rituel» : celui des «trois tours » autour de l'arbre précédemment implanté. En décidant plus tard d'instaurer ce rituel autour de l'arbre dans le contexte de l'esclavage, l'intention du roi était non seulement de matérialiser les "derniers adieux » vis-à-vis des femmes, des hommes et des enfants esclaves, mais aussi de respecter un tant soit peu la tradition vodun qui défend l'immortalité de l'âme ou de l'esprit de l'homme. C'est ainsi à partir d'enjeux religieux que la sacralisation de l'arbre a eu lieu. Le kigelia africana était devenu un dispositif ultime pour le Retour à la case de départ et pour le repos des âmes des individus maltraités, trahis par leurs propres frères noirs et forcés de partir loin de leurs proches avec des inconnus et pour des destinations inconnues. C'est à ce moment qu'il prit le nom de l'arbre du Retour.

7 Il faut souligner que dans les cultures indigènes béninoises dont le vodun, l'invisible occupe une place importante dans l'appréhension du monde. Selon ces cultures indigènes, les êtres humains doivent sans cesse entrer en contact avec les forces 
invisibles qui composent le monde surnaturel afin d'essayer de se concilier avec la puissance ou la bienveillance. Le vodun lui-même représente un culte pour le monde de l'invisible ${ }^{5}$. Au cours de ce culte, le prêtre vodun implore le dieu surnaturel de l'ouverture des portes des deux mondes, visible et invisible. Par ailleurs, d'après ces cultures indigènes l'âme doit "dormir en paix ». Dans le contexte de l'esclavage, le repos de l'âme n'est possible que si le rituel des «trois tours » est respecté par l'esclave avant sa déportation. Dans le cas contraire, l'âme vagabonderait sans un repos effectif.

C'est dans le cadre du premier festival mondial des arts et cultures vodun dénommé "Ouidah 92 ", organisé du 8 au 18 février 1993, que le peintre, modeleur et sculpteur béninois Cyprien Tokoudagba (1939-2012) a proposé une statue représentant l'arbre du Retour. Cette statue a été installée devant l'arbre dans les années 1993. D'après le récit de l'artiste, la statue représente la divinité « Aziza ». « Aziza » est l'esprit de la forêt. On considère que cette divinité est immatérielle et sans adeptes. Selon la Direction de la Culture et du Patrimoine de la Mairie de Porto Novo, la divinité « Aziza » est avant tout le symbole de la migration des peuples Houèdonou Hêvinou dans la commune des Aguégués (au Bénin). La divinité assure au sein de cette communauté la fonction de protection. Mais elle est aussi mise au service d'autres communautés et intervient dans ces cas pour le règlement des mœurs sociales ${ }^{6}$.

Aujourd'hui, l'arbre du Retour est communément appelé Hounti en langue locale Fon. Ce qui signifie l'arbre du sang. Il représente le lieu où se tiennent les danses cérémoniales des Egouns (revenants en langue locale Yoruba). Les Egouns sont des divinités à travers lesquelles on évoque l'esprit des morts. Les deux images ci-dessous représentent respectivement l'arbre du Retour et la divinité Egoun.

Image 2 : L'arbre du Retour, Route de l'Esclave, Ouidah, Bénin

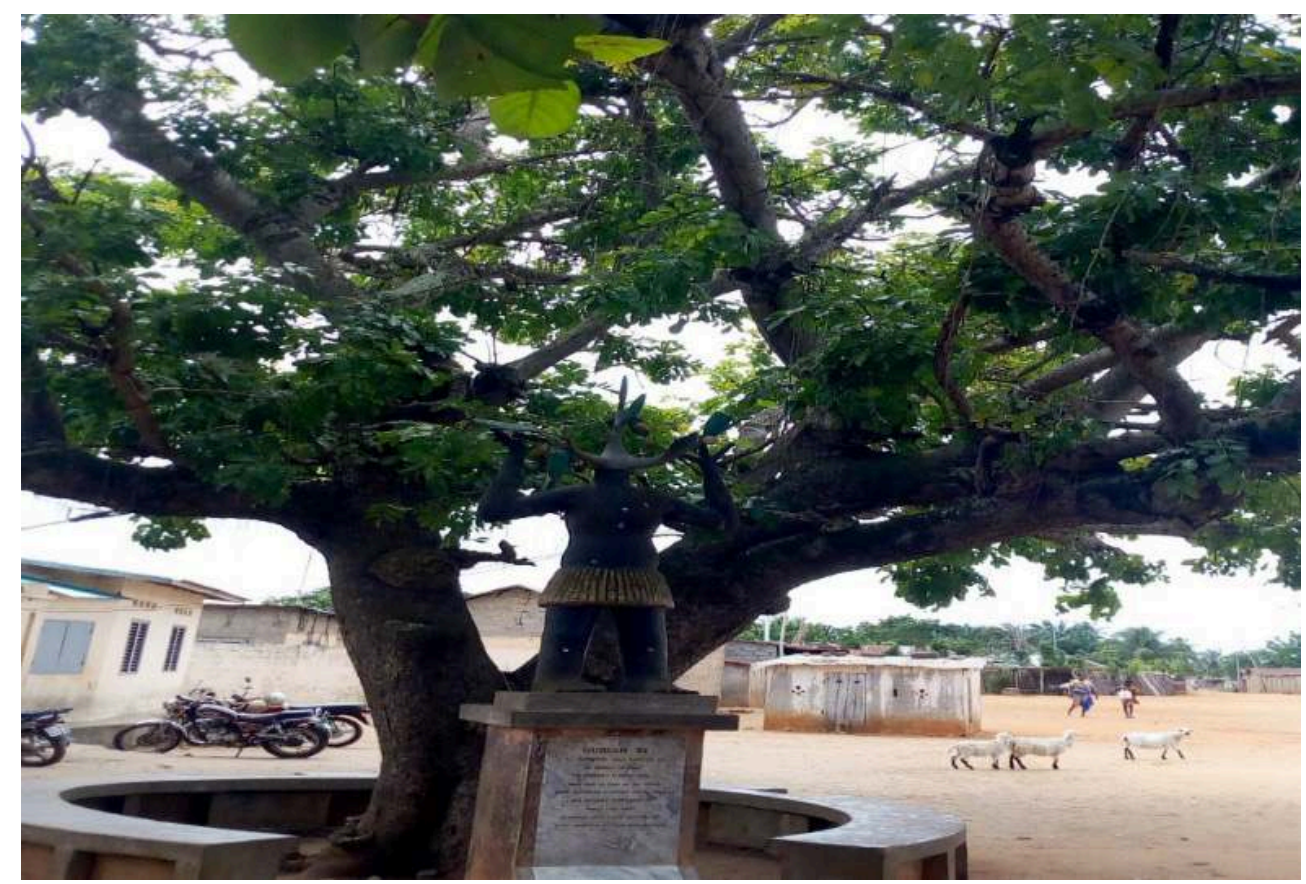

Photo prise dans le cadre de nos enquêtes 
Image 3 : La divinité Egoun (Revenant)

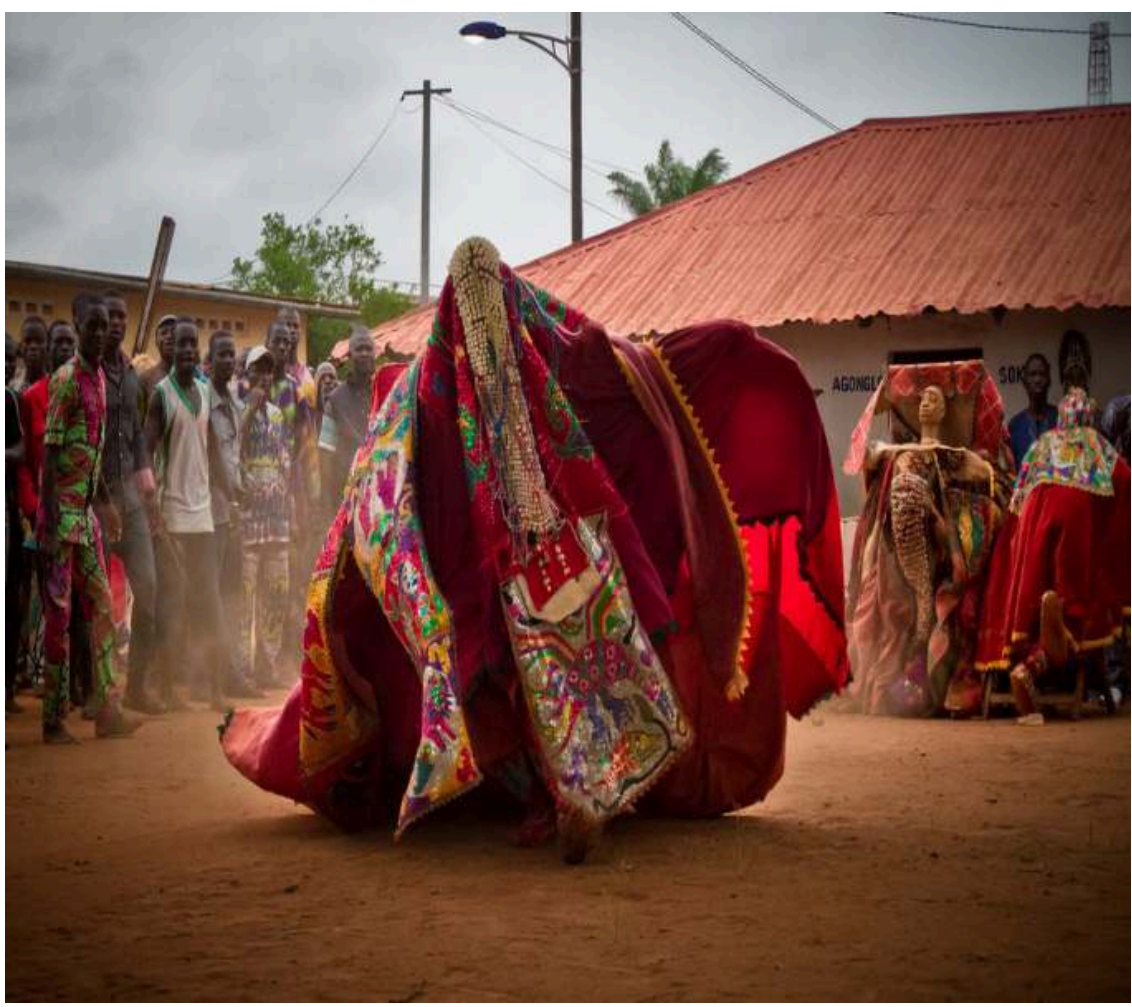

Journal hebdomadaire Adjinakou, Bénin. Photo prise à l'adresse : https://www.journal-adjinakoubenin.net/hebdo/societe/benin-interdiction-de-sortie-des-egoun-goun-les-motivations-du-prefettoboula/attachment/egungun1024x667/, consulté le 3 janvier 2020.

\section{Pratiques et institutions de la mort et du Retour dans la communauté de Ouidah à l'époque esclavagiste}

\section{Qu'entendons-nous par « pratique » et par « institution » ?}

Nous entendons attribuer à la notion de "pratique » le sens que lui donne Emmanuel Kant. La pratique, c'est « la mise en œuvre d'une certaine fin dont on peut considérer qu'elle observe certains principes de conduite qu'elle se représente d'une manière universelle. $»^{7}$ En considérant pratique et tactique, Karl Marx affirmait qu'on ne peut dissocier les pratiques liées à la structuration du réel et propres à une classe sociale, et les tactiques. Ces dernières, en effet, clarifient les processus par lesquels la société en question tente de s'«imposer» en tant que telle. De son côté, Florent Coste parle d'indexicalité. En s'appuyant sur l'exemple de l'utilisation d'un recueil de sermons, ce chercheur affirme qu'il doit consister moins en une " démarche stratégique » qu'en un "matériau de tactique ». C'est celui-ci qui permet une adaptation aux situations ${ }^{8}$.

Le mot institution dérive du verbe "instituer». Ce verbe renvoie dans tous les cas à l'acte d'établir ou celui de fonder quelque chose de manière constante. De ce fait, on peut définir l'institution comme étant l'établissement ou la fondation de quelque chose de manière constante. De plus, pour que l'institution soit effective, il faut avoir deux éléments que sont : la classe instituée, et les forces, les engagements etc., qui vont agir sur la chose instituée pour la faire exister réellement et surtout de façon continue. C'est 
ce que l'on peut lire à travers la définition de Lagroye et Offerlé (2010) de l'institution. D'après ces deux chercheurs donc, l'«institution peut être considérée comme une forme de rencontre dynamique entre ce qui est institué sous forme de règles, de modalités, de savoirs, etc. et les investissements (ou engagements) dans une institution qui seuls la font exister concrètement $"$.

12 Cette approche qui s'appuie sur la participation de l'individu nous semble la plus adaptée pour expliquer les pratiques rituelles liées à la mort et au Retour spirituel dans la communauté de Ouidah durant l'esclavage. En effet, nous épousons l'idée selon laquelle les pratiques sociales sont des construits (Crozier et Friedberg, 1997). «Il faut rompre avec toute vision déterministe de la conduite humaine pour le remplacer dans son contexte d'indétermination et de liberté relative, ce qui veut dire l'analyser comme l'expression d'un choix nécessitant certaines capacités de la part de celui - ou de ceux -

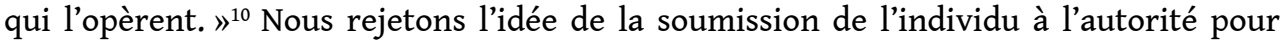
soutenir l'existence des contextes et dispositions qui le prédisposent à la soumission/ adhésion à l'institution. Comme l'avait souligné Jacques Chevallier, « les ressortissants (d'une institution) ne sont pas une cire molle dans laquelle s'inscrit identiquement l'empreinte de l'institution $»^{11}$.

\section{Pratiques et rites de l'institution de la mort}

13 Avant de continuer, nous allons expliciter la notion de "rites d'institution ». C'est une notion préférée par Bourdieu en 1982 au lieu et place des "rites de passages ». Alors que les rites de passage ont pour fonction de marquer une étape dans la vie d'un individu (souvent sur le plan social ou sexuel), les rites d'institution eux, ont pour fonction de légitimer, c'est-à-dire de «faire méconnaître en tant qu'arbitraire et reconnaître en tant que légitime, naturel ${ }^{12}$. Le rite en lui-même dispose d'un effet inaperçu majeur : celui de traiter différemment les hommes et les femmes. À cet effet, Bourdieu écrivait que le rite consacre la différence. «Il l'institue, instituant du même coup l'homme en tant qu'homme, c'est-à-dire circoncis, et la femme en tant que femme, c'est-à-dire non justifiable de cette opération rituelle. ${{ }^{13}}^{13} \mathrm{En}$ un mot, les rites constituent une distinction légitime de fait. Et le rituel d'institution constitue un acte de consécration, c'est-à-dire un acte qui consiste à sanctifier en faisant connaître et reconnaître une distinction préexistante ou non.

Dans le contexte de l'esclavage et dans la communauté de Ouidah, les esclaves ayant perdu leur liberté avaient pour devoir de suivre leur maitre. L'esclave n'avait plus de famille. Sa vie se résumait à l'obéissance à son maître. D'ailleurs sa condition physique exprimait bien sa vie d'esclave. En effet, l'esclave était privé de sa liberté par des chaînes autour de son cou, de ses poignets et de ses chevilles.

La mère de son voisin trouvait que la garde d'honneur sous la porte de HAN Qinhu était somptueuse, comparable à celle du roi, elle était curieuse et interrogea, l'un parmi eux répliqua : «je viens pour accueillir le roi. »; très vite, la garde d'honneur disparut. L'autre jour, un homme sévèrement malade, paniqué, vint devant la porte de Qinhu, et demanda de voir le roi, les gardiens demandèrent : «quel roi ? ", « le roi Yanluo » dit-t-il, les gardiens allaient le châtier, HAN Qinhu les arrêta et dit : « je suis parvenu à devenir le pilier du pays en vivant, et si je devenais le roi Yanluo, je n'aurais plus de regret. " et après, il fut malade au lit pour quelques jours et mourut.

Mais dans cette aventure, l'esclave perdait aussi sa masculinité ou sa féminité. Nous dirons tout simplement que l'esclave perdait sa valeur d'humain. D'abord, il était 
considéré tout au long du processus comme un objet. Il était troqué contre des objets de pacotille. Et il était marqué au fer rouge après avoir été acheté. 1 pipe faisait valoir 5 esclaves, 1 bouteille d'alcool donnait droit à 10 esclaves, et 1 canon faisait bénéficier de 15 hommes ou 21 femmes. Ensuite, l'esclave acceptait sa mort anticipée. La mort anticipée est antérieure. Elle concerne l'identité, l'histoire et la culture. Elle survenait lorsque l'esclave mâle achevait de faire le neuvième tour d'un arbre appelé l'arbre de l'Oubli. Du côté de la femme, cette mort anticipée et intériorisée survenait quand elle achevait de faire le septième tour de cet arbre. L'objectif de ce rituel chez les deux sexes était de faire d'eux des êtres sans aucune mémoire. Ainsi, ils ne risquaient pas de se rebeller. Le rituel des neuf tours (les hommes) ou sept tours (les femmes) est donc institué pour manifester l'effacement et la disparition de toute vie passée. Enfin, les esclaves subissaient la séquestration pendant trois à quatre mois dans une chambre obscure.

\section{Pratiques et rites de l'institution du Retour}

Après l'Oubli, le Retour spirituel devient important. C'est ainsi que le Roi Agadja décida du rituel des trois tours autour d'un autre arbre afin que l'esprit se retourne et trouve « la paix éternelle » sur cet arbre et auprès des siens. Tous les esclaves ont procédé à ce rituel sans aucune résistance. Nous pensons que cette obéissance collective constitue non seulement un fait de communication mais aussi un rite d'institution. Ces deux faits vont bien entendu ensemble. Ils contribuent tous les deux à montrer l'identité, la différenciation, la spécificité des religions indigènes. En fait, si dans toutes les religions la mort est un phénomène réel, ce qui se passe après ne fait pas l'unanimité. Certains parlent de réincarnation, alors que d'autres parlent du repos éternel de l'âme. Et pour d'autres encore, il ne se passe rien après la mort. Selon la principale religion indigène qui est le vodoun, ce qui reste à l'individu après sa mort, c'est son âme, son esprit. De telle sorte que les âmes ou les esprits des morts devenaient les gardiens des vivants. Où habitent ces esprits? Simplement dans l'environnement : comme l'avait écrit Birago Diop, les morts ne sont pas sous terre, ils sont dans le feu, dans le rocher, dans les herbes et dans la forêt ${ }^{14}$.

La mère de son voisin trouvait que la garde d'honneur sous la porte de HAN Qinhu était somptueuse, comparable à celle du roi, elle était curieuse et interrogea, l'un parmi eux répliqua : «je viens pour accueillir le roi. »; très vite, la garde d'honneur disparut. L'autre jour, un homme sévèrement malade, paniqué, vint devant la porte de Qinhu, et demanda de voir le roi, les gardiens demandèrent : «quel roi ? ", « le roi Yanluo » dit-t-il, les gardiens allaient le châtier, HAN Qinhu les arrêta et dit : «je suis parvenu à devenir le pilier du pays en vivant, et si je devenais le roi Yanluo, je n'aurais plus de regret. " et après, il fut malade au lit pour quelques jours et mourut.

Nous pensons en effet que l'acte des trois tours peut être considéré comme rite institué grâce à des éléments contextuels dont la participation individuelle. En d'autres termes, comment la construction sociale de ce rite des trois tours se fait-elle? Les premiers éléments que nous voulons exploiter pour expliquer ceci, ce sont les chaînes. Mais ces chaînes à elles seules ne justifient pas d'après nous la décision de participer. À celles-ci, nous pensons qu'il faut ajouter la contribution de l'individu en tant qu'agent individuel. Cette participation, Giddens l'avait pensée lorsqu'il écrivait ceci : «Dans des relations sociales entre êtres humains, les seuls objets mobiles sont les agents individuels qui utilisent des ressources pour que des choses se produisent, de façon intentionnelle ou 
autre. Les propriétés structurelles des systèmes sociaux "n'agissent pas sur" une personne de manière à la "forcer" à se comporter d'une façon particulière comme le feraient des forces de la nature. $»^{15}$

Que retenir de l'évolution des idéologies sur deux objets aussi incontournables que l'arbre de l'Oubli et l'arbre du Retour dans l'histoire du Bénin quatre siècles après ?

\section{Entre le Retour et la Présence : les représentations, les usages et les pratiques de communication autour de l'arbre de l'Oubli et de l'arbre du Retour aujourd'hui}

\section{Les représentations et les usages}

17 Nous avons procédé par des focus groupe pour collecter les discours de 24 personnes qui ont bien voulu nous accorder leur attention. Nous avons choisi de mener avec deux groupes de 12 personnes des entretiens semi-directifs et avons choisi d'opter pour une observation directe non participante. Les personnes interrogées sont âgées de 31 ans à 60 ans. Ils sont des commerçants, des prêtres religieux, et des guides touristiques. Tous ont une résidence aux alentours de l'arbre du Retour. Pour la plupart des personnes rencontrées, ce sont des résidences familiales. Comment justifier le choix de cette technique d'enquête? Les théories des représentations sont souvent associées à l'analyse du discours (AD). Elles se penchent sur l'avantage des méthodes qualitatives au détriment de méthodes quantitatives. C'est dans cette logique que l'entretien est considéré comme un outil pertinent et une méthode lisible, compréhensible et capable de fournir des informations sur l'évolution d'une représentation sociale (Lilian Negura, 2006). Perçu sous cet angle, l'entretien est le cadre où émerge le discours, principal support pour analyser les représentations (Moscovici, 1976) ${ }^{16}$. De son côté, l'analyse du discours appelle à un double objectif: d'abord, elle doit tenter de comprendre la subjectivité de l'énoncé, c'est-à-dire sa signification selon l'émetteur. Ensuite, elle doit comprendre l'objectivité de l'énoncé, c'est-à-dire sa pertinence pour le récepteur ou pour le groupe social en question.

18 C'est aussi pour cette raison que l'analyse du discours est convenable pour l'étude des représentations sociales car ces dernières «enracinent le discours dans un contexte symbolique familier pour les deux participants classiques de la communication » (Lilian Negura, 2006) ${ }^{17}$. Pour soutenir ce même fait, Francine Mazière écrit dans son livre consacré à l'analyse du discours ce qui suit: «l'AD ne sépare l'énoncé ni de sa structure linguistique, ni de ses conditions de production, historiques et politiques, $\mathrm{ni}$ des interactions subjectives, ni des préconstruits qui contraignent le sens. C'est à l'intérieur de ce programme qu'elle définit ses procédures de lecture. ${ }^{18} \mathrm{Il}$ apparaît dans nos analyses des discours que les représentations sociales de l'arbre du Retour s'organisent avant tout autour de "la mort». Mais nous observons que cette connotation ne définit plus à elle seule les représentations de l'arbre. La "mort " a évolué aujourd'hui et semble coexister avec la "présence». Mais coexiste-t-elle effectivement avec la " présence » ou la remplace-t-elle ? L'analyse thématique ne nous a pas permis de répondre effectivement à cette question. Ce que nous avons remarqué c'est que le contenu des discours porte essentiellement sur la "présence " plutôt que sur le «retour». En effet, l'arbre du Retour symbolise aujourd'hui aux yeux des enquêtés la croissance ou la perpétuité. Ce qui signifie déjà que l'histoire ne sera jamais 
éradiquée. Ici l'arbre est gage du renouvellement perpétuel de toute l'histoire de l'esclavage au Bénin.

19 Mais l'arbre du Retour s'inscrit également dans le fait social. Il incite à la bonne moralité.

Les bruits que les branches de l'arbre du Retour émettent sont un véritable instrument pour jauger l'honnêteté des usagers de cette place devenue aujourd'hui publique. « On a peur d'y rester si on n'est pas franc, si on est de moralité douteuse ». La sensation que les esprits des esclaves sont encore présents sur les lieux contraint les femmes et les hommes à avoir des pensées positives dans cet espace : « On ne profère pas des paroles négatives à l'endroit de quelqu'un sous cet arbre ; sinon on a le retour soi-même (retour à l'envoyeur). »

\section{L'arbre de l'Oubli et l'arbre du Retour comme espaces de communication}

L'arbre du Retour est un espace de règlements de compte entre des individus. C'est un lieu approprié pour traiter des mécontentements, des différends entre les individus, jeunes et vieux, à propos de tous les jours. Sous l'arbre on peut aussi tenir des réunions dans le cadre de l'organisation d'un événement concernant le village. Il est de même possible de discuter à propos d'autres sujets le plus souvent au cours de simples causeries entre frères et amis. Les moments de fréquentation de l'arbre sont variés : l'aube, le matin, midi, l'après-midi, le soir, très tard dans la nuit. Il n'existe aucune interdiction quant à l'heure de fréquentation de la place.

21 Un autre usage de l'arbre du Retour est lié aux incantations. Les constituants de l'arbre du Retour sont utilisés dans les cérémonies de sortie de l'enfant dans la famille royale des Kakanakou et des Dossougoin. On s'en sert aussi pour traiter certaines maladies. Par ailleurs, il faut noter certaines idées fausses à propos de l'arbre du Retour. Comme par exemple l'idée selon laquelle les esprits des hommes qui sont ensevelis empêchent un individu de réaliser un projet lorsque celui-ci le conçoit sous l'arbre du Retour. Ou bien l'idée selon laquelle on peut utiliser les constituants de l'arbre pour envouter quelqu'un (notamment l'éléphantiasis, le gonflement des pieds).

\section{Conclusion}

L'arbre est un objet important quand on aborde le sujet de la mort dans l'histoire du Bénin en général et dans l'histoire de l'esclavage du pays en particulier. C'est un objet susceptible d'accueillir le Retour des esprits des individus partis en esclavage, une fois qu'ils sont morts. Ce Retour spirituel nécessaire, selon les croyances indigènes, n'est effectif qu'à l'issue de deux rituels dont l'un est consacré à l'Oubli et donc à l'effacement ou la mort, et l'autre au Retour. Aujourd'hui, plusieurs siècles après l'abolition de l'esclavage, ces lieux historiques réservés respectivement à la mort et aux morts sont l'objet de représentations et d'usages sociaux, notamment l'arbre du Retour. 


\section{NOTES}

1. Nicole Loraux, La cité divisée - L'oubli dans la mémoire d'Athènes, Paris, Éditions Payot \& Rivages, avril 2005, p. 165.

2. Ambroise Tournyol du Clos, «La colonisation est-elle responsable des malheurs de l'Afrique », Conflits, hors-série $\mathrm{n}^{\circ}$ 3, Printemps 2016.

3. Gaetano Ciarcia, «Rhétoriques et pratiques de l'inculturation », Gradhiva, 8, 2008, p. 43.

4. Selon notre traduction à partir du fongbé, langue nationale béninoise.

5. https://fr.wikipedia.org/wiki/Vaudou.

6. Nous avons retenu cette définition à partir de la fiche réalisée par la Direction de la Culture et du Patrimoine de la Mairie de Porto Novo dans le cadre du projet « PaCTE - Patrimoine, Culture et Tourisme Équitable». Accessible sur http://patrimoinebenin.org/index.php/rechercheimages/1494-vodoun-aziza-rituels-lies-a-la-divinite-aziza-aire-s-culturelle-s-adja-tado, consulté le 3 novembre 2019.

7. Emmanuel Kant, «Sur le lieu commun : il se peut que ce soit juste en théorie, mais en pratique cela ne vaut rien », Théorie et pratique, trad. F. Proust, Paris, Flammarion, 1994, p. 45.

8. Florent Coste, « Pratiques rituelles et pragmatique de la conversion dans les sermons de sanctis du XIII siècle ", Revue tracès, Pratiques et Tactiques, nº 7, Lyon, 2004-2005.

9. Jacques Lagroye, Michel Offerlé (dir.), Sociologie de l'institution, Belin, 2010, p. 12.

10. Michel Crozier, Erhard Friedberg, L'acteur et le système, Paris, Le Seuil, 1977, p. 206.

11. Jacques Chevallier, «L'analyse institutionnelle », L'institution, Paris, PUF, 1981, p. 24.

12. Pierre Bourdieu, «Les rites comme actes d'institution », Actes de la recherche en sciences sociales, Rites et fétiches, vol. 43, juin 1982, p. 68.

13. Ibid., p. 68-69.

14. Diop Birago, Le souffle des ancêtres, recueil leurres et lueurs, ED, Présence Africaine, 1960.

15. Anthony Giddens, La constitution de la société. Éléments de la théorie de la structuration, Paris, PUF, 1987, p. 239.

16. Serge Moscovici, La psychanalyse, son image, son public, Paris, PUF, 1976, p. 38.

17. Lilian Negura, «L'analyse de contenu dans l'étude des représentations sociales ", Sociologies [Online], Theory and research, Online since 22 October 2006, connection on 29 October 2019. URL : http://journals.openedition.org/sociologies/993.

18. Francine Mazière, L'analyse du discours, Paris, PUF, 2005, p. 9.

\section{RÉSUMÉS}

Cet article étudie l'institution de la mort et du retour de l'âme dans l'histoire de l'esclavage au Bénin. Il montre comment les rituels traditionnels liés à l'esclavage sont autant de pratiques, mais aussi de rites d'institution de la mort et du retour. Il montre précisément la participation de l'esclave dans cette institution, de même que les sens et les représentations des habitants de ces espaces réservés à la mort et aux morts, plusieurs siècles après l'esclavage.

This study is about the institution of death and the return of the soul in benin's slavery history. It shows how the traditionals rituals of slavery are not only practices, but also rites of the 
institution of death and return. It shows precisely the participation of the slave in this institution, as well as the inhabitants's senses and representations regarding this spaces reserved for death and dead, several centuries after slavery.

INDEX

Mots-clés : L'Oubli et le Retour, pratiques, rites d'institution, la mort, Bénin

Keywords : Oblivion and return, pratices, rites of institution, death, Benin

\section{AUTEUR}

\section{CAROLE FAGADÉ}

MICA EA 4426, Université Bordeaux Montaigne

carole.fagade@etu.u-bordeaux-montaigne.fr 